Dossiê: Mundialização, regionalização e fronteiras

\title{
Regionalismo latino-americano Produção de saber e criação e importação de teoria
}

\author{
Latin American regionalism \\ Knowledge production and theory creation and import \\ Regionalismo latinoamericano \\ Producción de saber y creación e importación de teoría \\ (1) José Briceño Ruiz* \\ Philippe De Lombaerde**
}

\begin{abstract}
Resumo: Este artigo explora a existência de vozes e ideias latino-americanas a respeito da integração regional e avalia se essas vozes contribuíram para a teorização do regionalismo/integração regional. Da mesma forma, o artigo visa contribuir para a discussão a respeito da exportabilidade das teorias de integração regional, em particular, para analisar se as ferramentas teóricas desenvolvidas no estudo da integração regional na Europa são relevantes para explicar e entender os processos que acontecem na América Latina e no Caribe e mesmo além.
\end{abstract}

Palavras-chave: Integração regional. Regionalismo. Teoria. América Latina. União Europeia.

Abstract: This paper explores the existence of Latin American voices and ideas on regional integration and evaluates if those voices have contributed to the theorizing of regionalism/regional integration. Similarly, the paper aims to contribute to the discussion on the exportability of regional integration theories, in particular, if the theoretical tools developed in the study of regional integration in Europe are of any relevance to explain and understand the processes that have taken place in Latin America and the Caribbean or even beyond it.

Keywords: Regional integration. Regionalism. Theory. Latin America. European Union.

Resumen: Este artículo explora la existencia de voces e ideas latinoamericanas sobre integración regional y evalúa si esas voces contribuyeron a la teorización del regionalismo/integración regional. Igualmente, el artículo pretende contribuir a la discusión respecto a la exportabilidad de las teorías de la integración regional, en particular, si las herramientas teóricas desarrolladas en el estudio de la integración regional en Europa son relevantes para explicar y entender los procesos que ocurren en América Latina y el Caribe y más allá.

Palabras clave: Integración regional. Regionalismo. Teoría. América Latin. Unión Europea.

\footnotetext{
* Universidad Cooperativa de Colombia (Bogotá, Colômbia).

** Neoma Business School (Rouen, França).

autores.dados_biográficos
}

Civitas, Porto Alegre, v. 18, n. 2, p. 262-284, maio-ago. 2018

Exceto onde especificado diferentemente, a matéria publicada neste periódico

é licenciada sob forma de uma licença

Creative Commons Attribution-NonCommercial-NoDerivatives 4.0 International License 


\section{Introdução}

Neste artigo apresentamos algumas reflexões respeito do papel das teorias e da teorização nos debates e estudos sobre o regionalismo latinoamericano. Argumenta-se que a teorização que está enraizada num contexto regional é essencial para uma compreensão profunda e relevante do fenômeno. Ao mesmo tempo, propõe-se uma avaliação matizada da importação de teoria. Usar o conhecimento e a teorização específicos do contexto e do caso não é incompatível com o uso de quadros teóricos mais gerais que permitam o trabalho comparativo entre regiões e o fluxo de conhecimento inter-regional.

Este trabalho tem dois objetivos. Por um lado, explora-se a existência de vozes e ideias latino-americanas sobre a integração regional e, em particular, busca-se verificar se essas vozes contribuíram para a teorização do regionalismo/integração regional. Por outro lado, o artigo visa contribuir para a discussão sobre a exportabilidade ou transferência das teorias de integração regional. Em outras palavras, o artigo propõe-se a avaliar se as ferramentas teóricas desenvolvidas no estudo da integração regional na Europa (cada vez mais questionadas ou criticadas na região) são relevantes para explicar e compreender os processos que acontecem na América Latina e no Caribe, e além.

Na primeira seção do artigo se explora até que ponto a América Latina tem sido um gerador de ideias (propostas teóricas) sobre regionalismo/ regionalização/integração regional. Em seguida, se examina o tema da importação das teorias europeias na América Latina. Na terceira seção, distingue-se entre teorias como ferramentas analíticas e teorias como conjuntos normativos de ideias ou prescrições políticas. Na quarta seção, discute-se a importância da teoria europeia como uma caixa de ferramentas políticas. Finalmente, são tecidas algumas considerações finais.

\section{A teorização latino-americana do regionalismo}

Nesta parte avaliam-se as contribuições latino-americanas, muitas vezes ignoradas, para a construção de uma teoria da integração. Para tanto, adota-se uma compreensão não estrita de teorização. A América Latina tem, de fato, uma longa tradição de iniciativas de integração regional, que levou ao desenvolvimento de um pensamento integracionista. Desde os primeiros movimentos de independência, a ideia de unidade regional foi impulsionada por figuras fundadoras da história latino-americana como Simón Bolívar, José Cecilio del Valle ou Bernardo de Monteagudo. A ideia de unidade regional 
também esteve presente no século 19 e início do século 20 nas propostas de pensadores como Lucas Alamán, Juan Bautista Alberdi, Justo Arosemena, José María Samper Agudelo, Benjamín Vicuña Mackenna, José Martí, Eugenio María de Hostos, Manuel Ugarte, Fernando García Calderón ou Salvador Mendieta (Rivarola Puntigliano, 2012; Briceño Ruiz, 2012). No entanto, esses pensadores não desenvolveram teorias explicativas gerais, mas contribuíram com um "saber". Além disso, a maioria de suas propostas estavam associadas a um contexto regional no qual existiam temores a respeito da perda da independência ou da autonomia política vis-à-vis forças externas. Em consequência, a integração regional foi percebida principalmente como um processo político baseado na ideia de que uma base cultural similar levaria os estados latino-americanos a unificar esforços para enfrentar ameaças externas. A integração econômica foi secundária, mesmo que algumas propostas de acordos de livre comércio e navegação fossem promovidas em congressos hispano-americanos ao longo do século 19. Essa falta de interesse pela integração econômica regional foi ainda aprofundada após 1870, quando começou o chamado período de desenvolvimento orientado para o exterior ou período primário exportador na história econômica latino-americana (Bulmer Thomas, 2003).

Depois da segunda década do século 20, a ideia de integração econômica como mecanismo para fomentar o desenvolvimento regional gradualmente ganhou proeminência sobre as ideias de unidade política. A figura de Alejandro Bunge simboliza esse processo. Este economista argentino propôs a criação de uma união aduaneira sul-americana em 1909. Esta proposta foi relançada em 1926 por Bunge, pelo chileno Eliodoro Yáñez e por Guillermo Subercaseux (Bunge, 1926; Yáñez, 1926; Subercaseux, 1926). Isso foi seguido, em 1940, pela proposta do economista argentino Federico Pinedo de criar uma união aduaneira entre a Argentina e o Brasil. Os ministros das Relações Exteriores dos dois países, Enrique Ruiz Guiñazú (Argentina) e Oswaldo Aranha (Brasil), apoiaram esta proposta e o Tratado de União Aduaneira foi assinado em novembro de 1940 (Sanjuan, 1998).

Outras propostas de integração regional surgiram nas décadas de 1940 e 1950. Um projeto para criar a Organização Econômica da Grande Colômbia foi aprovado pela "Carta de Quito" em 1948 (Briceño Ruiz, 2013). As iniciativas de integração política e econômica regional tiveram impulso na década de 1950. Do lado político, líderes como o argentino Juan Domingo Perón na Argentina, Getúlio Vargas no Brasil e Carlos Ibañez no Chile tentaram reativar o Tratado ABC (Reckziegel, 1996). A integração econômica regional foi um dos pilares da nova estratégia de desenvolvimento. A Comissão Econômica para 
a América Latina (Cepal) e Raúl Prebisch conceberam a integração regional como um mecanismo para implementar uma estratégia de industrialização

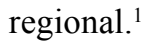

Assim, se se analisar a longue durée (Braudel, 1958) das ideias e propostas latino-americanas a respeito da integração regional, o início do processo com certeza se encontra bem antes da década de 1950. Em termos gerais, a maioria das iniciativas regionais é o resultado de um processo histórico de longo prazo que tem procurado, por um lado, atingir a autonomia política e, por outro lado, o desenvolvimento econômico e a industrialização regional (Briceño Ruiz, 2013). Isso levou à geração de um "saber" foucaultiano, ou seja, ideias e discursos que certamente não constituíam teorias formais, mas que proporcionavam uma estrutura na qual uma teoria poderia ser desenvolvida. Este "saber" latino-americano sobre a integração regional, desenvolvido principalmente antes de 1949, é uma fonte essencial para entender a lógica atual do regionalismo latino-americano.

Contudo, é justo reconhecer que o período moderno de integração regional na América Latina começou na década de $1950 \mathrm{com}$ as propostas lançadas por Prebisch e pela Cepal para a negociação um Mercado Comum Latino-Americano e da Associação Latino-Americana de Livre Comércio (Alalc), em 1960. Essas iniciativas foram acompanhadas por tentativas de desenvolver uma explicação formal do regionalismo latino-americano, inclusive com a construção de categorias teóricas. $\mathrm{Na}$ esfera econômica, o estruturalismo latino-americano proporcionou essa construção teórica. $\mathrm{Na}$ esfera política, esse papel foi desempenhado pela Escola da Autonomia, ligada a Helio Jaguaribe, no Brasil, e Juan Carlos Puig, na Argentina.

Embora seja acertado falar a respeito de uma "Escola estruturalista latino-americana", também deve ser reconhecido que essas ideias não podem ser vistas desconectadas dos escritos de autores como Hirschman, Kaldor, Kuznets ou Myrdal e de ideias similares emergentes em outros países (Ranis, 2004; Moncayo et al., 2011, p. 361). Para os estruturalistas, a integração econômica foi um elemento de uma estratégia geral para promover o objetivo de alcançar níveis mais altos de desenvolvimento econômico através de um processo endógeno de industrialização. Este processo visava reduzir a vulnerabilidade externa dos países em desenvolvimento e considerava o problema da deterioração a longo prazo dos termos de troca conhecida como

\footnotetext{
${ }^{1}$ No resto do texto, usaremos o acrônimo Eclac (ou seu equivalente espanhol, Cepal). Vale ressaltar, no entanto, que a Comissão Econômica das Nações Unidas para a América Latina foi criada em 1948; somente em 1984 foi adotada uma resolução, mudando o nome para a Comissão Econômica das Nações Unidas para a América Latina e o Caribe (UN-Cepal).
} 
a tese de Prebisch-Singer (Prebisch, 1950; Singer, 1950; Toye e Toye, 2003). A industrialização permitiria a absorção dos excedentes de oferta de mão-de-obra no setor rural, levaria a mudanças tecnológicas e a uma maior produtividade e melhoraria a distribuição da renda (Fitzgerald, 1998). Os estruturalistas referiam-se, assim, a um conceito integrado de desenvolvimento (Rodríguez, 2006; Moncayo, 2012, p. 29) que ainda caracteriza o pensamento latinoamericano atual de centro-esquerda sobre a regionalização, regionalismo e integração regional.

Raúl Prebisch foi uma figura central neste processo ao argumentar que a integração econômica regional foi um dos pilares da nova estratégia de desenvolvimento que ele promoveu como Secretário Executivo da Cepal. Segundo ele, os padrões de comércio observados desde meados do século 19 até meados do século 20 não confirmavam a teoria da vantagem comparativa, uma vez que os ganhos do comércio tinham sido divididos de forma desigual entre os países industriais (centro) e os estados agrícolas (periferia). O progresso tecnológico que elevou a produtividade ocorreu principalmente no centro, enquanto fatores estruturais domésticos e a divisão internacional do trabalho inibiram a periferia de se beneficiar desse avanço. Essa distribuição desigual do progresso técnico foi uma das causas dos preços cíclicos das matériasprimas e influenciou a demanda relativa de produtos industriais e produtos primários, levando ao declínio constante nos termos de troca da periferia. Essa deterioração afetou a possibilidade de desenvolvimento econômico ao reduzir a capacidade da periferia de comprar bens e serviços do centro. Para superar esta situação, Prebisch recomendou promover a industrialização pela via da substituição de importações, da proteção tarifária e dos incentivos aos investimentos estrangeiros no setor das manufaturas (Prebisch, 1996 [1949], 1950; Cepal, 1994).

No entanto, Prebisch criticou as políticas autárquicas de industrialização impulsionadas por vários países latino-americanos desde a década de 1920, no âmbito de mercados nacionais nos quais as economias de escala não foram necessariamente atingidas e a elevada proteção tarifária favoreceu o estabelecimento de fábricas ineficientes. Neste contexto, a integração econômica regional foi pensada como um dos pilares da estratégia de desenvolvimento econômico. A Cepal e Prebisch propuseram o desenvolvimento de um plano de industrialização regional, baseado na especialização no quadro de um mercado comum, para criar fábricas capazes de produzir bens de capital em condições competitivas e gerar economias de escala e sublinharam que o processo de integração regional não deveria ser autárquico nem hostil aos fluxos de investimento estrangeiro direto (IED); o objetivo a longo prazo era 
criar melhores condições para a integração da América Latina na economia mundial (Ecla, 1959; Tavares, 1998).

Essas ideias tiveram um papel importante na concepção e aplicação das políticas de integração na América Latina nas décadas de 1950, 1960 e 1970. Os Tratados de Manágua (1960) que estabeleceu o Mercado Comum CentroAmericano (MCCA) e de Cartagena (1969) que criou o Grupo Andino, por exemplo, mostraram claramente a influência das propostas da Cepal (Moncayo et al., 2011, p. 363-364). ${ }^{2}$ A integração foi concebida como um mecanismo para promover o desenvolvimento econômico regional, mas também como um processo para superar as restrições estruturais endógenas e exógenas que impediam o desenvolvimento econômico. ${ }^{3}$

No âmbito político, uma "teoria da autonomia", estreitamente ligada à integração regional, foi desenvolvida pelo brasileiro Helio Jaguaribe e pelo argentino Juan Carlos Puig nas décadas de 1970 e 1980. Jaguaribe descreveu o sistema internacional como uma ordem hierárquica, no qual alguns países são mais "autônomos" do que outros. Assim, Jaguaribe percebia o sistema internacional como um espaço diferenciado em quatro níveis, nos quais existiam várias hierarquias de poder (primazia geral, primazia regional, autonomia $\mathrm{e}$ dependência), todos caracterizados por patamares distintos de integridade territorial e autodeterminação (Jaguaribe, 1980). Para Jaguaribe, a autonomia depende de condições estruturais, que identificou como "viabilidade nacional" e "permissibilidade internacional". Jaguaribe também argumentou que dois fatores adicionais eram necessários para a existência de autonomia nos países periféricos: autonomia tecnológica e empresarial e relações favoráveis com o centro. Puig também criticou a teoria tradicional das relações internacionais, especificamente a abordagem realista. Para ele, o sistema internacional não era anárquico, mas sim hierárquico e organizado em torno de três tipos de atores: "distribuidores supremos" que são autônomos no seu processo de tomada de decisões, "distribuidores inferiores" que executam as instruções dos distribuidores supremos e os "destinatários", atores "que basicamente obedecem às decisões dos distribuidores supremos" (Puig, 1980). Para Puig,

\footnotetext{
${ }^{2}$ Há um debate sobre se a Associação Latino-Americana de Livre Comércio (Alalc), estabelecida pelo Tratado de Montevidéu em 1960, foi realmente influenciada pelas ideias de Prebisch e da Cepal. Contrariamente ao MCCA e ao Pacto Andino, a Alalc não incluiu um plano de industrialização regional. Somente se referia a acordos de complementação industrial, a serem negociados pelos estados interessados. Na biografia de Dosman sobre Prebisch (Dosman, 2008), mostra-se que o último não estava muito satisfeito com o que foi acordado no Tratado de Montevidéu.

${ }^{3}$ Sobre a evolução (e racionalização) subsequente do pensamento da Cepal na política comercial e integração regional na direção do chamado regionalismo aberto, ver Cepal (1994) e Pizarro (1999). Ver também Moncayo (2012) e Moncayo et al. (2011).
} 
a integração regional devia basear-se na solidariedade e devia promover mecanismos para melhorar a viabilidade nacional e, consequentemente, o posicionamento político da América Latina na ordem internacional estratificada (Puig, 1987).

Assim, uma explicação precisa do processo complexo do regionalismo latino-americano contemporâneo deve considerar esse acervo composto tanto pelo "saber" pré-1949 quanto pelas teorias formais acima referidas, em particular para entender a busca persistente de desenvolvimento econômico e de autonomia política que tem marcado a história dos países e da integração latino-americanos. O trabalho de vários especialistas em regionalismo latino-americano hoje, incluindo os trabalhos respeito do "regionalismo pós-hegemônico", também podem estar situados nesta tradição intelectual estruturalista/autonomista (Tussie e Riggirozzi, 2012; Briceño Ruiz e Morales, 2017).

Pode-se argumentar ainda que o regionalismo comercial nas Américas pode ser entendido também a partir alguns novos conceitos, como modelo de "hub and spoke" (centro e raio) e "spaguetti bowl" (prato de espaguete), desenvolvidos no contexto da proliferação de acordos comerciais nas Américas desde a década de 1990, mais ou menos hierarquicamente centrados nos Estados Unidos, que caracterizou o chamado "novo regionalismo" de inspiração neoliberal (IDB, 2002; De Lombaerde e Garay, 2008). Este foi o resultado combinado, por um lado, da reorientação da política comercial dos Estados Unidos na década de 1990, e por outro lado, do papel ativo desempenhado por países como o México e o Chile na negociação desses acordos. Depois da criação do Tratado de Livre Comércio da América do Norte (Tlcan ou Nafta em inglês) em 1992, o México assinou acordos similares com Costa Rica, Colômbia e Venezuela no âmbito do chamado Grupo dos Três (G-3) e com El Salvador, Guatemala e Honduras no quadro do chamado "Triângulo do Norte", além de uma série de acordos extra-regionais. Do mesmo modo, o Chile assinou acordos comerciais com os Estados Unidos, Canadá, México, Colômbia, Venezuela, Bolívia, Peru, Equador, Panamá, Costa Rica e o Mercado Comum Centro-americano. O resultado é conhecido como um "spaguetti bowl" (Bhagwati, 1995), o que se refere à coexistência complexa de acordos de comércio bilaterais (intra ou extra) ou regionais parcialmente justapostos, que causam custos de transação não negligenciáveis para os agentes econômicos (Wonnacott, 1975; 1982; 1996). O modelo de "hub and spoke" contribui para a compreensão analítica dos efeitos econômicos (e até certo ponto políticos) de uma rede de acordos comerciais centrada em uma economia central ou "hub" (os Estados Unidos neste caso) 
e que inclui "sub-hubs" (como Chile e México) e "raios" (Wonnacott e Wonnacott, 1996).

Finalmente, pode-se argumentar que a tese inter-presidencialista sobre a dinâmica do regionalismo no Mercosul e, mais amplamente, na América do Sul também é uma contribuição de estudiosos latino-americanos para a teorização enraizada no contexto regional (Malamud, 2005).

\section{Importância da teoria europeia: caixa de ferramentas analítica}

Na seção anterior, avaliou-se em que medida América Latina foi uma plataforma para a geração de "saber" e teorias respeito do regionalismo. Contudo, como se articula isso com o predomínio das teorias europeias, como referido anteriormente? Isto é considerado nas seções a seguir. A este respeito, geralmente não se faz distinção entre duas questões distintas: a primeira é se as teorias europeias (ou seja, teorias originalmente desenvolvidas para explicar o processo de integração europeia) podem ser exportadas como ferramentas analíticas, em vez de teorias normativas, para investigar o regionalismo latino-americano. A segunda é em que medida os quadros e modelos teóricos e normativos europeus têm influenciado de fato na formulação de políticas regionais na América Latina. Quando se diz que as teorias não podem ser exportadas, os autores muitas vezes misturam as duas questões ou, pelo menos, não explicitam a que se referem. Nesta seção, analisa-se a primeira questão; a segunda será analisada na próxima.

Se as teorias europeias podem ser exportadas como ferramentas analíticas para outras regiões, depende de duas condições: a primeira condição é que os vários casos de regionalismo (latino-americano, europeu e outros) ou seus aspectos sejam exemplos de uma categoria comum. A segunda condição é que as teorias sejam suficientemente abstratas.

Os regionalismos latino-americanos e europeus pertencem a uma categoria comum? Esta condição refere-se à dimensão ontológica na construção da teoria e está relacionada a uma questão fundamental (existencial) no regionalismo comparativo, também conhecido como o "problema $\mathrm{n}=1$ " (De Lombaerde et al., 2010). A questão é saber se o caso da integração europeia e, por extensão, todos os outros casos, é um caso único, sui generis, ou se é apenas uma expressão concreta, específica de um contexto, de um fenômeno mais geral (integração regional, regionalismo e/ou regionalização) que é observada em várias regiões do mundo (Caporaso, 1997). Esta não é uma discussão nova no campo; já nas primeiras pesquisas sobre regionalismo havia uma consciência de que a comparabilidade era uma questão que merecia ser considera. Os autores que aderiram à abordagem dos subsistemas regionais 
eram geralmente favoráveis à comparação entre regiões. Thompson (1973, p. 91), por exemplo, argumentou que

[...] existe uma excelente oportunidade para obter mais informações através da análise comparativa. De que outra forma vamos apreender quais formas de comportamento são 'universalmente' regionais e quais são particulares de tipos específicos de região? Deste modo, deveria ser possível evitar os área-centrismos ou, pelo menos, saber onde eles são apropriados.

Para Deutsch et al. (1957, p. 15-19) a comparação nunca é perfeita, contextos geográficos e históricos específicos devem ser levados em consideração, mas a comparação não deve ser abandonada. No entanto, outros autores, como Nye (1968, p. 880), referiram-se ao caráter ambíguo e complexo da União Europeia e ao problema da comparabilidade dos processos de integração regional em diferentes regiões do mundo. Esta ideia da UE como uma política ad hoc ganhou gradualmente terreno quando as instituições europeias foram desenvolvidas (Keohane e Hoffmann, 1991; Hix, 1994, 1998; Caporaso, 1996; Kohler-Koch e Eising, 1999; Hooghe e Marks, 2001). Em consequência, alguns sugeriram que os sistemas políticos nacionais, especialmente os estados federalistas, poderiam ser melhores casos de comparação do que outros esquemas regionais menos avançados (Krislov, Ehlermann e Weiler, 1986; Sbragia, 1992; Hix, 1994). Os estudos da UE tornaram-se, em geral, um campo de estudo próprio, desenvolvido em grande medida independentemente do estudo de outros regionalismos.

Apesar deste desenvolvimento, destacados especialistas defenderam a tese da comparabilidade e enfatizaram o potencial da análise comparativa incluindo a UE (Marks, 1997; Pollack, 1997, Caporaso, 1997). Em essência, eles argumentam que a UE é uma polity, um sistema político híbrido, com certas características que justificam a comparação com outras regiões ou organizações regionais, mesmo que seja aceito que outras características, especialmente as relacionadas ao seu caráter supranacional, também permitam uma comparação relevante com os estados federais. Se a comparação faz sentido ou não, depende da pergunta de pesquisa específica que está sendo feita. Esta é também a nossa posição.

Dois comentários adicionais devem ser feitos respeito deste ponto. Primeiro, a admissão do recurso à experiência europeia na análise comparativa não implica que ela deva ser considerada como referência ou norma (voltaremos sobre este assunto na próxima seção). Em segundo lugar, na medida em que 
o contexto seja relevante/importante para entender o desenvolvimento do regionalismo em uma determinada região, esse contexto, ou seus aspectos mais importantes, pode ser incorporado na análise comparativa. Isto é o que é chamado de "comparação contextualizada". Mais recentemente, vários autores também argumentaram a favor da pesquisa comparativa sobre o regionalismo incluindo a UE (Ebbinghaus, 1998; Clarkson, 2000; Checkel, 2007; PostelVinay, 2007; De Lombaerde et al., 2009a; Warleigh-Lack e Rosamond, 2010; Söderbaum e Sbragia, 2010; Van Langenhove, 2012). De acordo com Warleigh-Lack e Van Langenhove (2010, p. 548-549), na discussão sobre a comparabilidade da UE, há muitas vezes uma confusão entre as abordagens nomotéticas e idiográficas. Eles sugerem "desempacotar" as regiões de acordo com a dimensão de sua "statehood" para que elas se tornem mais comparáveis (Warleigh-Lack e Van Langenhove, 2010, p. 549-553).

A segunda condição para a importação bem-sucedida de teorias europeias como teorias analiticamente relevantes é que elas sejam suficientemente abstratas. ${ }^{4}$ Para alguns analistas isso é evidente. Para Malamud (2010, p. 650), por exemplo, "falar de teorias da integração europeia é tão inadequado quanto falar de teorias da política alemã ou de partidos políticos estadunidenses: teorias não são estudos de caso, mas explicações sistemáticas de fenômenos gerais". No entanto, este entendimento não é consensual. A questão é saber se as "teorias europeias" são suficientemente abstratas. A resposta imediata é "sim"; é preciso lembrar que a teoria da integração regional dominante na primeira fase do pósguerra, ou seja, o neofuncionalismo, já tinha uma aspiração clara e explícita à teoria geral. Foi, no entanto, na década de 1960 que projetos de pesquisa em larga escala foram desenvolvidos para "testar" essa teoria em outras regiões do mundo (Haas; Schmitter, 1964; Haas, 1967).

Isso não significa que não houvesse consciência da possível natureza problemática de tal exercício, ou seja, o de construir uma teoria inspirada na observação de um caso e logo aplicá-la a outros casos aparentemente semelhantes. Em outros trabalhos, por exemplo, Haas manifestou algumas dúvidas e cautela a respeito da relevância e da aplicabilidade de sua abordagem em outras regiões do mundo e argumentou que não podia haver uma "lei de integração" universal "deduzida do exemplo europeu” (Haas, 1961, p. 289, 1976). Para Haas, é provável achar diferentes configurações de fatores explicativos em diferentes regiões que explicam os respectivos processos de integração regional, e atores e fatores extrarregionais que desempenham

\footnotetext{
${ }^{4}$ Para uma visão geral das "teorias da integração europeia", ver Rosamond (2000), Schwok (2005), Schimmelpfennig e Rittberger (2006), Wiener e Diez (2009), e Saurugger (2010).
} 
papéis distintos em tais experiências (Haas, 1961). Haas (1970, p. 628) afirmou mesmo que

a limitação [do neofuncionalismo] está inserida na fonte de sua abordagem - a política democrática pluralista-industrial moderna. Essa fonte oferece um raciocínio para vincular as diferentes variáveis que existem no modelo neofuncionalista na Europa Ocidental; mas sua aplicação ao Terceiro Mundo até agora só tem servido para prever com precisão as dificuldades e falhas na integração regional, enquanto no caso europeu uma bem-sucedida previsão positiva foi alcançada.

A respeito deste assunto, pode-se argumentar que, seguindo sua lógica, a aplicabilidade do modelo pode ser uma questão de tempo. Em um artigo posterior, ele apresentou uma visão mais matizada (Haas, 1976), argumentando que, embora fosse bem possível que a teoria da integração regional (neofuncionalista) não fosse mais aplicável a certas dimensões do processo de integração europeia, ainda era muito útil para avaliar os processos de integração regional na América Latina ou na Ásia. Ele destacou ainda que a continuidade da relevância dessas teorias dependia da existência de processos de tomada de decisão incrementais, por um lado, e de um sistema regional relativamente autônomo, por outro. Na década de 1970, o conceito neofuncionalista de spill over foi complementado com conceitos relacionados, tais como spill-back, spill-around e encapsulation (Schmitter, 1970a, 1970b). Isso tornou a teoria neofuncionalista mais geral e compatível com uma variedade de cenários no mundo real. Mais recentemente, Mattli (2005), Malamud e Schmitter (2007) e Jørgenson e Valbjørn (2012, p. 18-19), entre outros, argumentaram em favor da aplicabilidade do modelo neofuncionalista como ferramenta analítica fora da Europa. No entanto, nem todos os especialistas compartilham desta opinião. Para Acharya, por exemplo, "nenhuma dessas teorias funcionou bem quando foram aplicadas em áreas não-ocidentais" (Acharya, 2012, p. 7). Somos de opinião de que a teoria neofuncionalista possui certas características abstratas que, à primeira vista, pareceriam torná-la aplicável ou, pelo menos, testável além da UE. Isso não significa, no entanto, que esta teoria captura os processos dinâmicos em outras regiões. Porém, mesmo que sirva apenas para explicar por que certos mecanismos (como o "spill over" ou a "politização", que são, como tais, também conceitos suficientemente gerais) não são observados em outros contextos, já demonstra sua utilidade.

As teorias econômicas da integração regional (não somente as teorias comerciais, mas também as teorias sobre os bens públicos regionais e as áreas 
monetárias ótimas) parecem ainda menos problemáticas desde a perspectiva da sua abstração. Um bom exemplo da aplicação do quadro explicativo dos bens públicos regionais a um contexto latino-americano pode-se observar em Estevadeordal, Frantz e Nguyen (2002). Também existem vários, embora não sejam muitos, exemplos de aplicação da teoria das áreas monetárias ótimas e seus desenvolvimentos para a cooperação e integração monetária nas Américas e no Caribe..$^{5}$ A abordagem eclética de Mattli (1999) também foi aplicada no caso latino-americano (Hummel; Lohaus, 2012).

Algumas considerações finais são apropriadas neste ponto: a primeira é a existência de diferentes níveis de teorização, a que aludimos. Também na teoria da integração europeia, se tem produzido uma série de desenvolvimentos teóricos com diferentes profundidades, passando de modelos teóricos específicos sofisticados para tipificações ontológicas básicas. Outra consideração é que a teorização em torno da integração europeia é muitas vezes simplesmente uma aplicação de teorias mais gerais das ciências sociais (construtivismo social, economia política etc.). Portanto, se a teoria da integração europeia é aplicável ou não em outros contextos regionais requer uma resposta matizada e em níveis múltiplos. Não faz muito sentido descartar categorias ontológicas relativamente abstratas e simples, apenas porque foram inicialmente usadas para descrever aspectos do processo de integração europeia. Também não faz muito sentido descartar as teorias da integração europeia se elas são essencialmente aplicações de teorias ou abordagens mais gerais das ciências sociais.

\section{A influência dos modelos teóricos e normativos europeus sobre a formulação de políticas regionais na América Latina}

Tem sido repetido que o processo de integração europeia não deve necessariamente ser um modelo para a integração regional ou para projetos de cooperação em outras regiões. Esta é uma declaração política e normativa a qual subscrevemos. Gradualmente se tornou "politicamente correto" questionar o uso da UE como um "modelo", não só na América Latina (Breslin e Higgott, 2003; Breslin et al., 2002; Hurrell, 2007; Warleigh-Lack; Rosamond, 2010; Warleigh-Lack e Van Langenhove, 2010; Söderbaum e Sbragia, 2010). Higgott (2006, p. 23), por exemplo, critica o "status paradigmático [do projeto de integração europeia] [...] contra o qual todos os outros projetos são julgados".

\footnotetext{
${ }^{5}$ Ver, por exemplo, Bayoumi e Eichengreen (1994), Lalonde e St.-Amant (1995), Giffith (1998), Fernández Castro (1998), Eichengreen (1998), De Lombaerde (1999) e Giambiagi (1999). Mais recentemente (e com uma abordagem muito mais crítica) surgiram novas abordagens que incluem Fritz (2006) e Fritz e Mühlich (2010).
} 
Contudo, é possível que a percepção de que o uso inadequado da experiência europeia como um "modelo" seja mais um problema ao nível dos discursos políticos do que da pesquisa acadêmica, embora isso não seja consenso.

Uma questão distinta é saber se o projeto de integração europeia influenciou de fato a criação e institucionalização de projetos regionais em outros lugares, como na América Latina. Sobre isso, há extensa produção. (De Lombaerde e Schultz, 2009a; Botto, 2009; Briceño Ruiz; Rivarola Puntigliano, 2009; Caballero, 2009; Bustamante e Giacalone, 2009; De Lombaerde, Pietrangeli e Schulz, 2009). A influência pode ser direta ou indireta. A influência direta pode ser "suave" (soft) ou "dura" (hard). A primeira refere-se aos fluxos de ideias, a última, à promoção explícita pelas instituições europeias da integração regional em outras partes do mundo, geralmente ligada à disponibilidade de fundos específicos de cooperação para o desenvolvimento ao nível regional. A influência indireta pode adotar a forma de regionalismo estratégico e de efeitos dominó. Vale ressaltar que influência não precisa necessariamente ser "positiva" no sentido de emular, seguir, adaptar; ela pode ser "negativa" ou reativa, como podem ser as consequências da recente crise europeia (Acharya, 2012).

Pode ser bem documentado que a influência europeia é tão antiga quanto a integração regional da pós-guerra na América Latina. Nas propostas da Cepal, por exemplo, pode-se argumentar que se vê uma confluência de influências do quadro teórico estruturalista - por sua vez, ligado à teorização pós-colonial do desenvolvimento em outros lugares, por um lado, e do caso europeu em desenvolvimento e da teorização relacionada a ele, por outro. O modelo de integração proposto pela Cepal teve as seguintes características:

(i) um mercado comum aberto a todos os países latino-americanos, com uma tarifa externa comum e uma cobertura universal de produtos, a serem estabelecidos por etapas; (ii) a harmonização das políticas industriais e dos acordos industriais, ${ }^{6}$ a fim de incentivar uma fase avançada de substituição de importações; (iii) o tratamento especial para países relativamente menos desenvolvidos e para o setor agrícola tradicional; (iv) cláusulas de salvaguarda para produtos sensíveis e mecanismos de compensação para assegurar uma distribuição equitativa de benefícios; (v) um acordo multilateral de pagamentos; e (vi) uma integração estatal com um papel ativo para o setor privado" (Moncayo et al., 2011, p. 362-363).

\footnotetext{
${ }^{6}$ Isso implicou a ideia de planejamento industrial regional. Ver, por exemplo, os programas
} setoriais de desenvolvimento industrial (PSDI) no caso andino (Ffrench-Davis, 1974). 
Argumentou-se que Prebisch se inspirou na literatura neofuncionalista sobre a integração europeia (Moncayo et al., 2011, p. 364-365) para sua avaliação inicial e positiva da institucionalização do Grupo Andino, incluindo o estabelecimento de uma Secretaria Executiva (Prebisch, 1973, p. 34-35). Contudo, Prebisch não foi particularmente entusiasta com a ideia de supranacionalidade presente na experiência europeia. Para Prebisch,

Nem nas ideias originais que surgiram da Cepal nem na concretização dessas ideias em Montevidéu, qualquer referência à supranacionalidade é feita [...]. Tanto na marcha progressiva em direção ao mercado comum quanto na organização final da mesma, não é possível pensar que as decisões que afetam o desenvolvimento econômico e social de cada país membro devem ser subordinadas à regra da maioria, em detrimento da soberania de país algum (Prebisch, 1969, p. 2).

Os quadros conceituais e as teorias europeias influenciaram os debates, como o relacionado ao quadro institucional mais conveniente para o regionalismo latino-americano. Isso ocorreu nos Andes, onde a Comunidade Andina copiou o desenho institucional da União Europeia, estabelecendo uma Comissão, um Parlamento e um Tribunal de Justiça supranacional. Este quadro institucional foi rejeitado por alguns setores políticos que preferiam um modelo intergovernamental. $\mathrm{O}$ debate europeu entre o intergovernamentalismo e o supranacionalismo tem sido replicado no Mercosul, onde a ausência de instituições supranacionais é considerada, por alguns especialistas, como uma das razões da crise que este esquema regional tem experimentado nos últimos anos.

$\mathrm{Na}$ esfera econômica, o processo de integração europeia também tem sido, em alguns aspectos, um modelo para a América Latina desde a década de 1960. A lógica da integração econômica que se desloca de uma área de livre comércio para um mercado comum como apresentada por Balassa (1961) para explicar a experiência europeia foi imitada em esquemas regionais como o Pacto Andino, na década de 1970, ou o Mercosul, na década de 1990. Do mesmo modo, algumas iniciativas, como o Pacto Andino, compartilham com a experiência europeia um modelo econômico de integração regional em que o desenvolvimento industrial e a justiça social têm um papel a desempenhar. Esta é uma questão crucial: tanto na Europa como na América Latina, a integração regional não foi considerada como sinônimo de liberalização do comércio, visto apenas como um aspecto de um processo complexo mais amplo. Finalmente, a escola estruturalista francesa também foi influente na 
década de 1970 na América Latina, especialmente no que diz respeito às suas propostas para criar polos de integração econômica.

\section{Considerações finais}

O objetivo deste trabalho foi refletir sobre o papel da teoria na compreensão dos regionalismos latino-americanos. Argumenta-se que existe um acervo latino-americano a respeito do regionalismo. Também se avalia a possibilidade de um diálogo das teorias europeias com as propostas originadas na América Latina. Em particular, se discute se alguns aspectos das teorias europeias podem viajar para a América Latina. Mesmo que se considere que a integração europeia e latino-americana tem suas particularidades, compartilham também motivos comuns que permitem uma comparação. Argumenta-se que nos debates sobre o caráter geral das teorias de integração regional, é necessário distinguir entre sua capacidade analítica, por um lado, e suas características normativas e uso político, por outro. As conclusões deste trabalho podem ser resumidas da seguinte forma:

Em primeiro lugar, reconhecemos a especificidade do regionalismo latino-americano, bem como as características próprias do desenvolvimento das ideias sobre ele. Assim, argumentamos que, a partir de uma perspectiva histórica de longo prazo, pode-se observar a existência de uma antiga tradição de "saber" sobre a integração regional que remonta ao início do século 19. No entanto, foi apenas na década de 1950 que esse "saber" foi transformado em explicações teóricas (normativas) formais de integração regional na América Latina. Este foi o caso das ideias de Prebisch e da Cepal sobre a relação entre desenvolvimento econômico, industrialização e integração regional e as propostas de uma integração regional baseada na solidariedade formuladas pela Escola de Autonomia nos anos 1970 e 1980. Alguns aspectos da recente lógica "pós-hegemônica" da integração regional na América Latina respondem a esta tradição.

Em segundo lugar, se argumenta que, a despeito de seu próprio acervo sobre regionalismo, a América Latina também tem sido influenciada por experiências externas de integração econômica e política. Em particular, a experiência de integração europeia influenciou o regionalismo latino-americano em aspectos específicos, como a lógica da integração comercial, o quadro institucional e a preocupação com a equidade. Por consequência, argumentase que os debates teóricos europeus sobre o regionalismo influenciaram os debates latino-americanos e que as teorias europeias não são necessariamente irrelevantes para explicar aspectos da lógica da integração regional na América Latina. Contudo, leituras europeias a respeito da integração regional precisam 
ser contextualizadas, considerando a história e a geografia, quando sejam aplicadas ao caso latino-americano.

Isso nos leva à nossa terceira conclusão, a saber, que os quadros ontológicos e as abordagens teóricas europeias contêm uma parte importante do conteúdo suficientemente abstrato para serem potencialmente úteis e relevantes para o trabalho comparativo. Isso é verdade para categorias ontológicas básicas, mas também para abordagens como as do intergovernamentalismo, do intergovernamentalismo liberal ou do construtivismo, que se baseiam em premissas gerais que potencialmente poderiam ter poder explicativo em diferentes casos e contextos. Mesmo abordagens como o neofuncionalismo podem ser úteis para explicar por que outras regiões têm ou não tem seguido caminhos semelhantes aos da integração europeia.

No entanto, considerar que as teorias europeias podem explicar alguns aspectos da lógica da integração regional na América Latina e em outras partes do mundo, não implica aceitar a premissa ontológica que assimila a integração regional com a integração regional com supranacionalidade ao estilo europeu. Preferimos utilizar a categoria regionalismo como um conceito que apreende um processo complexo em que alguns espaços regionais geográficos promovem instituições nas quais o intergovernamentalismo e a supranacionalidade podem existir ou, mesmo, coexistir e que podem assumir diferentes formas e abranger diversas áreas políticas.

A questão de saber se as teorias podem viajar de uma região para outra é uma questão sobre a abstração das teorias. As teorias também têm níveis diversos; o desafio é extrair conceitos e mecanismos abstratos das teorias relacionadas ao contexto. Este conteúdo abstrato pode ser formulado de forma útil como hipóteses de pesquisa em projetos de pesquisa comparativa contextualizados.

Em suma, uma abordagem matizada e multifacetada é necessária para que exista possibilidade de uma pesquisa comparativa sobre o regionalismo e a possibilidade de construir quadros ontológicos e teóricos que tenham uma relevância para além de uma região específica, seja Europa ou América Latina. Isto não é incompatível com o reconhecimento da importância e do valor do contexto e da teorização local, ou regional; não é incompatível com o fato de evitar ficar preso em "gaiolas conceituais" ao pensar no futuro do regionalismo latino-americano.

\section{Referências}

ACHARYA, Amitav. Comparative regionalism: a field whose time has. Come? The International Spectator. v. 47, n. 1, p. 3-15, $2012<10.1080 / 03932729.2012 .655004>$. 
BALASSA, Bela. The theory of economic integration. London: Allen \& Unwin, 1961. BAYOUMI, Tamim; EICHENGREEN, Barry. Monetary and exchange rate arrangements for Nafta. Journal of Development Economics. v. 43, n. 1, p. 125-165, $1994<10.1016 / 0304-3878(94) 90026-4>$.

BHAGWATI, Jagdish. US trade policy: the infatuation with FTAs. In: Jagdish Bhagwati; Anne O. Krueger (orgs). The dangerous drift to preferential trade agreements. Washington: American Enterprise Institute, 1995. p. 1-18.

BOTTO, Mercedes I. The role of epistemic communities in the 'makability' of Mercosur. In: Philippe De Lombaerde; Michael Schulz (orgs.). The EU and world regionalism. The makability of regions in the 21st century. London: Ashgate, 2009. p. 171-185.

BRAUDEL, Fernand. Histoire et sciences sociales: la longue durée. Annales. Économies, sociétés, civilisations. v. 13, n. 4, p. 725-753, 1958.

BRESLIN, Shaun; HIGGOTT, Richard. New regionalism(s) in historical perspective. Asia-Europe Journal. v. 1, n. 2, p. 167-182, 2003.

BRESLIN, Shaun; HUGHES, Chris W.; PHILlIPS, Nicola; ROSAMOND, Ben (orgs.). New regionalisms in the global political economy: theories and cases. New York: Routledge, $2002<10.4324 / 9780203361672>$.

BRICEÑO RUIZ, José. Autonomía y desarrollo en el pensamiento integracionista latinoamericano. In: José Briceño Ruiz; Andrés Rivarola Puntigliano; Angel Casas Gragea (orgs.). Integración latinoamericana y caribeña. Madrid: Fondo de Cultura Económica, 2012. p. 27-58.

BRICEÑO RUIZ, José. The Andes: a long tradition of thought and practice about regional integration. In: Andrés Rivarola Puntigliano; José Briceño Ruiz (orgs.). The resilience of regionalism in Latin America and the Caribbean. Development and autonomy. Houndsmill: Palgrave, 2013. p. 146-182.

BRICEÑO RUIZ, José; RIVAROLA PUNTIGLIANO, Andrés. The European Union and the 'making' of South American regionalism. In: Philippe De Lombaerde; Michael Schulz (orgs.). The EU and world regionalism. The makability of regions in the $21 \mathrm{st}$ century. London: Ashgate, 2009. p. 101-114.

BRICEÑO RUIZ, José; MORALES, Isidro. Post-hegemonic regionalism in the Americas: toward a Pacific-Atlantic divide? Abingdon: Routledge, 2017.

BULMER THOMAS, Victor. The economic history of Latin America since independence. Cambridge: Cambridge University Press, $2003<10.1017 /$ CBO9780511817397>.

BUNGE, Alejandro. Manifiesto acerca de la situación económica actual de Europa. El manifiesto de los banqueros e industriales europeos. Revista de Economía Agentina. v. 102, n. 9, p. 469-487, 1926.

BUSTAMANTE, Marleny; GIACALONE, Rita. An Assessment of European Union Cooperation towards the Andean Community (1992-2007). In: Philippe De Lombaerde; Michael Schulz (orgs.). The EU and world regionalism. The makability of regions in the 21st century. London: Ashgate, 2009, p. 149-169. 
CABALLERO, José. Normative Europe: Changing values and attitudes in Central America. In: Philippe De Lombaerde; Michael Schulz (orgs.). The EU and world regionalism. The makability of regions in the 21 st century. London: Ashgate, 2009. p. 131-147.

CAPORASO, James A. The European Union and forms of the state: Westphalia, regulatory, or post-modern? Journal of Common Market Studies. v. 34, n. 1, p. 29-52, $1996<10.1111 / \mathrm{j} .1468-5965.1996 . t b 00559 . x>$.

CAPORASO, James A. Does the European Union represent an n of 1? ECSA Review. v. 10, n. 3, p. 1-2, 1997.

CEPAL. El regionalismo abierto en América Latina y el Caribe. La integración económica al servicio de la transformación productiva con equidad. Santiago de Chile: Naciones Unidas, 1994.

CHECKEL, Jeffrey T. Social mechanisms and regional cooperation: are Europe and the EU really all that different? In: Amitav Acharya; Alastair Iain Johnston (orgs.). Crafting cooperation. Regional international institutions in comparative perspective. Cambridge: Cambridge University Press, 2007. p. 221-243.

CLARKSON, Stephen. 'Apples and oranges'. Prospects for the comparative analysis of the EU and Nafta as Continental Systems. EUI Working Papers. n. 23, 2000.

DE LOMBAERDE, Philippe. Integración monetaria caribeña, choques simétricos y comercio intraindustrial. Innovar. n. 13, p. 176-177, 1999.

DE LOMBAERDE, Philippe; GARAY, Luis Jorge. El nuevo regionalismo en América Latina. In: Philippe De Lombaerde; Shigeru Kochi; José Briceño Ruíz (orgs.). Del regionalismo latinoamericano a la integración interregional. Madrid: Siglo XXI, 2008. p. 3-35.

DE LOMBAERDE, Philippe; PIETRANGELI, Giulia; SCHULZ, Michael. EU Support to Latin American regionalism. In: Christian Franck; Jean-Christophe Defraigne; Virginie de Moriamé (orgs.). The European Union and the rise of regionalism: source of inspiration and active promoter/L'Union européenne et la montée du régionalisme: exemplarité et partenariats. Brussels: Academia Bruylant, 2009. p. 247-261.

DE LOMBAERDE, Philippe; SCHULZ, Michael. The EU and world regionalism. The makability of regions in the 21st century. London: Ashgate, 2009.

DE LOMBAERDE, Philippe, SÖDERBAUM, Fredrik; VAN LANGENHOVE, Luk; BAERT, Francis. The problem of comparison in comparative regionalism. Review of International Studies. v. 36, n. 3, p. 731-753, $2010<10.1017 / \mathrm{S} 0260210510000707>$.

DEUTSCH, Karl W. et al. Political community and the North Atlantic area: international organization in the light of historical experience. Princeton: Princeton University Press, 1957.

DOSMAN, Edgar J. The life and times of Raùl Prebisch: 1901-1986. Montréal: McGill-Queen's Press, 2008.

EBBINGHAUS, Bernard. Europe through the looking-glass: comparative and multi-level perspectives. Acta Sociologica. v. 41, n. 4, p. 301-313, 1998 $<10.1177 / 000169939804100401>$. 
ECLA. The Latin American Common Market. New York: Undesa, n. E/CN.12/531, 1959.

EICHENGREEN, Barry. Does Mercosur need a single currency? Cider Paper. n. C98-103, 1998.

ESTEVADEORDAL, Antoni; FRANTZ, Brian; NGUYEN, Tam Robert (orgs.). Regional public goods: from theory to practice. Washington: IDB-ADB, 2002.

FERNÁNDEZ CASTRO, Rosanna. Una evaluación de los costos de una unión monetaria en el Mercosur. Revista de Economía. v. 5, n. 2, p. 33-49, 1998.

FFRENCH-DAVIS M., Ricardo. La planificación en el Pacto Andino y el arancel externo común. Revista de la Integración. n. 17, p. 87-104, 1974.

FITZGERALD, Valpy. La CEPAL y la teoría de la industrialización. Revista de la Cepal. Número especial, 1998.

FRITZ, Barbara. So far from god and so close to the US dollar: contrasting approaches of monetary coordination in Latin America. In: Barbara Fritz; Martina Metzger (orgs.). New issues in regional monetary coordination. London: Palgrave, 2006. p. $126-146$.

FRITZ, Barbara; MÜHLICH, Laurissa. South-South monetary integration: the case for a research framework beyond the theory of optimum currency area. International Journal of Public Policy. v. 6, n. 1-2, p. 118-135, 2010.

GIAMBIAGI, Fabio. Mercosur. Why does monetary union make sense in the long run? Ensaios BNDES. 1999.

GIFFITH, Winston. Integración monetaria en el Caricom. Innovar. n. 12, p. 135-151, 1998.

HAAS, Ernst B. International integration: the European and the universal process. International Organization. v. 15, n. 3, p. 366-392, $1961<10.1017 /$ S0020818300002198>.

HAAS, Ernst B. The uniting of Europe and the uniting of Latin America. Journal of Common Market Studies. v. 5, p. 315-345, $1967<10.1111 / \mathrm{j} .1468-5965.1967$. tb01153.x>.

HAAS, Ernst B. The study of regional integration: reflections on the joy and anguish of pretheorizing. International Organization. v. 24, n. 4, p. 607-646, 1970.

HAAS, Ernst B. Turbulent fields and the theory of regional integration. International Organization, v. 30, n. 2, p. 173-212, $1976<10.1017 /$ S0020818300018245>.

HAAS, Ernst B.; SCHMITTER, Philippe C. Economics and differential patterns of political integration: projections about unity in Latin America. International Organization, v. 18, n. 4, p. 705-737, $1964<10.1017 /$ S0020818300025297>.

HIGGOTT, Richard. The theory and practice of region. In: Bertrand Fort; Douglas Webber (org.). Regional integration in East Asia and Europe: convergence or divergence. London: Routledge, 2006.

HIX, Simon. The study of theEuropeanCommunity: the challengetocomparative politics. West European Politics. v. 17, n. 1, p. 1-30, $1994<10.1080 / 01402389408424999>$. 
HOOGHE, Liesbeth; MARKS, Gary. Multi-level governance and European integration. Boulder-Lanham: Rowman and Littlefield, 2001.

HUMMEL, Felix; LOHAUS, Mathis. Mercosur: integration through presidents and paymasters. In: Tanja A. Börzel; Lukas Goltermann; Mathis Lohaus; Kai Striebinger (orgs.). Roads to regionalism. Farnham: Ashgate, 2012. p. 59-77.

HURRELL, Andrew. One world? Many worlds? The place of regions in the study of international society?. International Affairs, v. 83, n. 1, p. 127-146, $2007<10.1111$ / j.1468-2346.2007.00606.x>.

IDB. Beyond Borders. The new regionalism in Latin America. Economic and social progress in Latin America 2002 Report. Washington: Inter-American Development Bank, 2002.

JAGUARIBE, Helio. Autonomia periférica e hegemonia cêntrica. Relações Internacionais. v. 3, n. 5, p. 8-24, 1980.

JØRGENSON, Knud Erik; VALBJØRN, Marten. Four dialogues and a funeral of a beautiful relationship: European studies and new regionalism. Cooperation \& Conflict. v. 47, n. 1 , p. $3-27,2012<10.1177 / 0010836711433105>$.

KEOHANE, Robert O.; HOFFMANN, Stanley (orgs.). The new European Community: decision making and institutional change. Boulder: Westview Press, 1991.

KOHLER-KOCH, Beate; EISING, Rainer (orgs.). The transformation of governance in the European Union. London: Routledge, 1999.

KRISLOV, Samuel, EHLERMANN, Claus-Dieter; WEILER, Joseph. The political organs and the decision-making process in the United States and the European Community. In: Mauro Cappelletti; Monica Seccombe; Joseph Weiler (orgs.). Integration through law: Europe and the American federal experience. v. 1: Methods, tools and institutions. Berlin: Walter de Gruyter, 1986.

LALONDE, René; ST.-AMANT, Pierre. Áreas de moneda optima: el caso de México y de Estados Unidos. Monetaria. n. 4, p. 431-459, 1995.

MALAMUD, Andrés. Presidential diplomacy and the institutional underpinnings of Mercosur: an empirical examination. Latin American Research Review. v. 40, n. 1, p. $138-164,2005<10.1353 /$ lar.2005.0004>.

MALAMUD, Andrés. Latin American regionalism and EU studies. Journal of European Integration. v. 32, n. 6, p. 637-657, $2010<10.1080 / 07036337.2010 .518720>$.

MALAMUD, Andrés; SCHMITTER, Philippe. The experience of European integration and the potential for integration in South America. Ibei Working Papers. n. 6, 2007.

MARKS, Gary. Does the European Union represent an n of 1? Ecsa Review. v. 10, n. 3, p. 2-3, 1997.

MATTLI, Walter. The logic of regional integration. Europe and beyond. Cambridge: Cambridge University Press, 1999.

MATTLI, Walter. Ernst Haas's evolving thinking on comparative regional integration: of virtues and infelicities. Journal of European Public Policy. v. 12, n. 2, p. 327-348, $2005<10.1080 / 13501760500044157>$. 
MONCAYO, Edgard; DE LOMBAERDE, Philippe; GUINEA IBÁÑEZ, Oscar. Latin American regionalism and the role of UN-Eclac, 1948-2010. In: Claude Auroi; Aline Helg (orgs.). Latin America 1810-2010. Dreams and legacies. London: Imperial College Press, 2011. p. 359-386.

MONCAYO, Edgard. The contribution of the regional UN Economic Commissions to Regional Integration Processes: the case of Eclac. In: Philippe De Lombaerde; Francis Baert; Tânia Felício (orgs.). The United Nations and the regions. Dordrecht: Springer, 2012. p. 27-49.

NYE, Joseph S. Comparative regional integration: concept and measurement. International Organization. v. 22, n. 4, p. 855-880, $1968<10.1017 / \mathrm{S} 00208183$ 00013837>.

PIZARRO, Ramiro. Comparative analysis of regionalism in Latin America and AsiaPacific. Serie Comercio Internacional. n. 6, Santiago de Chile: Cepal, 1999.

POLLACK, Mark. Does the European Union represent an n of 1?. Ecsa Review. v. 10, n. 3, p. 4-5, 1997.

POSTEL-VINAY, Karoline. The historicity of the international region: revisiting the 'Europe and the rest' divide. Geopolitics. v. 12, n. 4, p. 555-569, 2007 $</ 10.1080 / 14650040701546046>$.

PREBISCH, Raúl. The economic development of Latin America and its principal problems. Lake Success: United Nations, 1950.

PREBISCH, Raúl. La marcha hacia el Mercado Común Latinoamericano. Santiago de Chile: Ilpes, 1969.

PREBISCH, Raúl. La integración económica en América Latina. In: Eric WyndlamWhite. La integración latinoamericana en una etapa de decisiones. Buenos Aires: BID and Intal, 1973.

PREBISCH, Raúl. El desarrollo latinoamericano y algunos de sus principales problemas. El Trimestre Económico. v. 63-1, n. 249, p. 175-245, 1996 [1949].

PUIG, Juan Carlos. Doctrinas internacionales y autonomía Latinoamericana. Caracas: Fundación Bicentenario de Simón Bolívar, 1980.

PUIG, Juan Carlos. Latin American integration and international regime. Caracas: Universidad Simón Bolívar, 1987.

RANIS, Gustav. The evolution of development thinking: theory and policy. Economic Growth Center Discussion Paper. Yale University. n. 886, 2004.

RECKZIEGEL, Ana L. G. S. O Pacto ABC. As relações Brasil: Argentina na década de 1950. Passo Fundo: Ediupf, 1996.

RIVAROLA PUNTIGLIANO, Andrés. Tres vertientes del pensamiento integracionista en Latinoamérica. In: José Briceño Ruiz; Andrés Rivarola Puntigliano; Angel Casas Gragea (orgs.). Integración latinoamericana y caribeña. Madrid: Fondo de Cultura Económica, 2012. p. 79-102.

RODRÍGUEZ, Octavio. El estructuralismo latinoamericano. Mexico DF: Siglo XXICepal, 2006. 
ROSAMOND, Ben. Theories of European integration. Houndsmills: MacMillan, 2000.

SAURUGGER, Sabine. Théories et concepts de l'intégration européenne. Paris: SciencesPo Presse, 2010.

SANJUAN, Alfonso. Camino al Mercosur: antecedente poco conocido, Cuadernos de Marcha, v. 2, n. 141, p. 50-56, 1998.

SBRAGIA, Alberta M. Thinking about the European future: the uses of comparison. In: Alberta M. Sbragia (org.). Euro-Politics. Institutions and policy-making in the 'new' European Community. Washington DC: The Brookings Institution, 1992. p. 257-291.

SCHIMMELPFENNIG Frank; RITTBERGER, Berthold. Theories of European integration. assumptions and hypotheses. In: Jeremy Richardson (org.). European Union. Power and policy making. Oxford: Oxford University Press, 2006. p. 74-9.

SCHMITTER, Philippe. A revised theory of regional integration. International Organization. v. 24, n. 4, p. 836-868, 1970a <10.1017/S0020818300017549>.

SCHMITTER, Philippe. The process of Central American integration: spill-over, spillaround or encapsulation? Journal of Common Market Studies. v. 9, n. 1, p. 1-48, 1970b $<10.1111 / \mathrm{j} .1468-5965.1970 . t b 00031 . x>$.

SCHWOK, René. Théories de l'intégration européenne. Paris: Montchrestien, 2005.

SINGER, Hans. The distribution of gains between investing and borrowing countries. American Economic Review. v. 40, p. 473-485, 1950.

SÖDERBAUM, Fredrik; SBRAGIA, Alberta. EU studies and the 'new regionalism': what can be gained from dialogue? Journal of European Integration. v. 32, n. 6 , p. $563-582,2010<10.1080 / 07036337.2010 .518716>$.

SUBERCASEAUX, Guillermo. Notas sobre las uniones aduaneras. La Nación. Santiago de Chile, 30 Nov. 1926.

TAVARES, Maria da Conceição. La Cepal y la integración económica de América Latina. Revista de la Cepal. número extraordinario, p. 213-228, 1998.

THOMPSON, William R. The regional subsystem: a conceptual explication and a propositional inventory. International Studies Quarterly. v. 17, n. 1, p. 89-117, 1973 $<10.2307 / 3013464>$.

TOYE, John F.J.; TOYE, Richard. The origins and interpretation of the PrebischSinger thesis. History of Political Economy. v. 35, n. 3, p. 437-467, 2003.

TUSSIE, Diana; RIGGIROZZI, Pia. The rise of post-hegemonic regionalism: the case of Latin America. Dordrecht: Springer, 2012.

VAN LANGENHOVE, Luk. Why we need to 'unpack' regions to compare them more effectively. The International Spectator. v. 47, n. 1, p. 16-29, 2012 $<10.1080 / 03932729.2012 .655005>$.

WARLEIGH-LACK, Alex; ROSAMOND, Ben. Across the EU studies - new regionalism frontier: invitation to a dialogue. Journal of Common Market Studies. v. 48, n. 4, p. $993-1013,2010<10.1111 /$ j. 1468-5965.2010.02083.x>. 
WARLEIGH-LACK, Alex; VAN LANGENHOVE, L. Rethinking EU studies: the contribution of comparative regionalism. Journal of European Integration. v. 32, n. 6 , p. $541-562,2010<10.1080 / 07036337.2010 .518715>$.

WIENER, Antje; DIEZ, Thomas (orgs.) European Integration Theory. 2. ed. Oxford: Oxford University Press, 2009.

WONNACOTT, Ronald J. Canada's future in a world of trade blocs: a proposal. Canadian Public Policy. v. 1, n. 1, p. 118-130, $1975<10.2307 / 3549842>$.

WONNACOTT, Ronald J. Controlling trade and foreign investment in the Canadian economy: some proposals. Canadian Journal of Economics. v. 15, p. 574-576, 1982.

WONNACOTT, Ronald J. Trade and investment in a hub-and-spoke system versus a free trade area. The World Economy. v. 19, n. 3, p. 237-252, $1996<10.1111 /$ j.14679701.1996.tb00676.x>.

WONNACOTT, Ronald J.; WONNACOTT, Paul. El Tlcan y los acuerdos comerciales en las Américas. In: Paul Krugman et al. Las Américas: integración económica en perspectiva. Bogotá: DNP-BID, 1996. p. 103-136.

YAÑEZ, Eliodoro. Hacia la unión aduanera y monetaria de América Latina. La Nación. Santiago de Chile, 9-11 nov. 1926.

Recebido em: 18 dez. 2017

Aprovado em: 27 mar. 2018

Autor correspondente:

José Briceño Ruiz

Carrera 9 no. 64-43, Edificio Casiquiare Apto. 301 - Chapinero

Bogotá, D.C., Colombia

JOSÉ BRICEÑO RUIZ <bricenoruiz@hotmail.com>

Doutor em Ciência Política pelo Institut d'études politiques d'Aix-en-Provence (Aix em Provence, França), professor na Universidad Cooperativa de Colombia (Bogotá, Colômbia).

ORCID: http://orcid.org/0000-0003-2710-4435

PHILIPPE DE LOMBAERDE <philippe.de-lombaerde@neoma-bs.fr>

Doutor pela Rheinisch-Westfälische Technische Hochschule Aachen (RWHT Aachen, Aachen, Alemanha), professor Associado de Economia Internacional na Neoma Business School (Rouen, França).

ORCID: http://orcid.org/0000-0002-6546-6771 